\title{
Elastic moduli of soils dependent on pressure: a hyperelastic formulation
}

\author{
G. T. HOULSBY*, A. AMOROSI† and E. ROJAS $\ddagger$
}

\begin{abstract}
The elastic behaviour of granular materials is non-linear, in that the small-strain tangent stiffness depends on the stress level. The elastic moduli typically vary as power functions of the mean stress. Simple models of this nonlinearity can result in behaviour that violates the laws of thermodynamics. To guarantee that an elasticity model is thermodynamically acceptable it must be possible to derive the elastic behaviour from a free energy potential (or alternatively from a complementary energy potential). In this paper elasticity models are derived that allow for variation of elastic moduli as power functions of mean stress, while guaranteeing thermodynamic acceptability. The important issue of the dependence of secant stiffness on strain amplitude (a phenomenon related to dissipation processes in the soil) is acknowledged but not addressed here.
\end{abstract}

KEYWORDS: anisotropy; elasticity; stiffness; stress
Le comportement élastique des matières granulaires est non linéaire, en ce que la rigidité tangente de petite déformation dépend du niveau de contrainte. De manière typique, les modules élastiques varient en fonction de la contrainte moyenne. Des modèles simples de cette non linéarité peuvent montrer des comportements qui vont à l'encontre des lois thermodynamiques. Pour garantir qu'un modèle d'élasticité est acceptable sur le plan de la thermodynamique, il doit être possible de dériver le comportement élastique d'un potentiel d'énergie libre (ou d'un potentiel d'énergie complémentaire). Dans cet exposé, nous dérivons des modèles d'élasticité qui tiennent compte de la variation des modèles élastiques en tant que fonctions de la puissance de la contrainte moyenne tout en garantissant le respect de la thermodynamique. L'importante question de la dépendance de la rigidité sécante par rapport à l'amplitude de la déformation (phénomène lié aux processus de dissipation dans le sol) est soulignée ici mais pas discutée.

\section{INTRODUCTION}

In recent years a considerable amount of experimental research has been carried out to investigate the mechanical behaviour of soils undergoing very small strains, for which the response is usually assumed to be reversible. The interpretation of the data shows that the initial soil stiffness (also known as small-strain stiffness) is a non-linear function of the stress (specifically the mean effective stress). The stiffness is also affected by other variables, such as the voids ratio, and/or the preconsolidation pressure.

Analysis of many geotechnical problems depends on a realistic representation of the non-linear dependence of the initial stiffness on stress. This is most often achieved by adopting hypoelastic formulations (Fung, 1965) in elasticplastic models, in which varying tangent moduli are defined. For instance, it is common to adopt the following procedure to calculate elastic moduli for the Modified Cam-Clay model. The bulk modulus $K$ is usually defined through the pressure-dependent expression $K=p^{\prime}(1+e) / \kappa$, and the shear modulus $G$ is then obtained by assuming a constant Poisson's ratio $v$. Such a model leads to non-conservative elastic response (Zytynski et al., 1978). This means that (for instance) multiple cycles applied to such a material could lead to continuous production of energy, which is clearly physically incorrect. A numerical model employing such an approach for analysis of cyclic behaviour might therefore lead to totally unreasonable results. The above approach also introduces a mild elastic-plastic coupling (discussed below). An alternative is to adopt the hyperelastic approach, which

Manuscript received 22 April 2004; revised manuscript accepted 24 February 2005.

Discussion on this paper closes on 1 December 2005, for further details see $p$. ii.

* Department of Engineering Science, Oxford University, UK

$\dagger$ Department of Civil and Environmental Engineering, Technical University of Bari, Italy.

† Universidad Autónoma de Querétaro, Facultad de Ingeniería (Posgrado), Mexico. is based on the existence of an energy potential, from which the reversible response can be derived. This naturally leads to a conservative elastic response, guaranteed to obey the First Law of Thermodynamics, and thus avoiding the problems on cycling described above. We assume here that it is self-evident that compliance with the laws of thermodynamics should be a required feature of any soil model to be used in geotechnical calculations: conversely a model that does not embody such a compliance is known ab initio to be flawed. Even for monotonic loading problems, in which the problems of an unconservative model may not be so apparent, there seems little justification for using an approach that is known to be thermodynamically inadmissible, when an acceptable alternative is available. It is difficult to quantify the differences between the models described below and the unconservative approach using a constant Poisson's ratio: for some load cases they will indeed be small, but areas where there might be significant differences would include: (a) behaviour over many cycles, in which an unconservative model could lead to spurious strain accumulation; and (b) cases where shearing at relatively large shear stresses would involve stress-induced anisotropy in a conservative model, which would not be predicted by the unconservative approach.

It is worth remarking that it is well recognised that the soil stiffness is also significantly dependent on the strain amplitude. This raises more difficult problems of hysteresis and energy loss. Strain-amplitude-dependent moduli are not addressed further here, although it is recognised that dependence on strain amplitude is at least as important as dependence on stress, and in many applications can indeed be more important.

Several models that address concerns about thermodynamic acceptability have been developed to reproduce the reversible behaviour of sands (e.g. Vermeer, 1978; Boyce, 1980; Lade \& Nelson, 1987), and others were aimed at describing the elasticity of clays (Houlsby, 1985; Hueckel et al., 1992; Borja et al., 1997; Niemunis \& Cudny, 1998). However, not all of the above models are characterised by 
energy recovery along closed stress or strain paths, and the models that do not have this property are thus not correctly formulated in a thermodynamic sense (Niemunis \& Cudny, 1998). Furthermore, some of these models assume a purely linear dependence of the stiffness on effective stress (i.e. $G$ directly proportional to $p^{\prime}$ ), whereas many soils exhibit a non-linear relationship.

The objective of this work is to present a hyperelastic, isotropic energy potential capable of accounting for the nonlinear dependence of the elastic stiffness on the stress. We first review briefly some typical experimental observations of the small-strain stiffness of soils, and their semi-empirical interpretations. This is followed by an introduction to linear hyperelasticity and its generalisation to the non-linear case, with particular reference to triaxial conditions. An isotropic energy potential function is then proposed, and its major features are explored in both triaxial and general stress conditions. An extension of the proposed potential is also provided, to account for the effects of the overconsolidation ratio (or alternatively preconsolidation pressure) on the initial stiffness of clays. Predictions from the proposed hyperelastic formulation are compared with bender element test results obtained on a reconstituted clay, compressed along radial stress paths. Finally some remarks are made on the consequences of the elastic-plastic coupling, introduced in the formulation for clays.

The standard soil mechanics sign convention of compressive stresses and strains positive is assumed, and all stresses are effective stresses (the prime notation being omitted for brevity). The following notation is adopted: $\sigma_{i j}$ is the effective Cauchy stress tensor; $\varepsilon_{i j}$ is the small-strain tensor; and $\delta_{i j}$ is the Kronecker delta $\left(\delta_{i j}=1\right.$ if $i=j, \delta_{i j}=0$ if $\left.i \neq j\right)$. We use the summation convention over a repeated index, so that

$$
\sigma_{i i} \equiv \sum_{i=1}^{3} \sigma_{i i}
$$

The stress invariants are $p=\frac{1}{3} \sigma_{i i}, q=\sqrt{ }\left(\frac{3}{2} s_{i j} s_{i j}\right)$, where $s_{i j}$ $=\sigma_{i j}-p \delta_{i j}$ is the deviatoric component of the effective stress tensor. The corresponding strain invariants are $v=\varepsilon_{i i}$ and $\varepsilon=\sqrt{ }\left(\frac{2}{3} e_{i j} e_{i j}\right)$, where $e_{i j}=\varepsilon_{i j}-\frac{1}{3} v \delta_{i j}$ is the deviatoric component of the strain tensor. For triaxial conditions $p=\frac{1}{3}\left(\sigma_{1}+2 \sigma_{3}\right), q=\sigma_{1}-\sigma_{3}, v=\varepsilon_{1}+2 \varepsilon_{3}, \varepsilon=\frac{2}{3}\left(\varepsilon_{1}-\varepsilon_{3}\right)$.

\section{SELECTED EXPERIMENTAL EVIDENCE}

Most of the published experimental data on the smallstrain stiffness of soils are derived from dynamic laboratory tests on samples of natural or reconstituted clays, or reconstituted sands, in triaxial conditions and under isotropic stress states. The data are principally derived either from resonant column equipment or from measurements using bender elements fitted (usually) vertically in triaxial systems. As a consequence, the small-strain shear stiffness $G_{0}$ has received the most attention in the literature. In the following all references to the shear modulus refer to the small-strain modulus, for which we shall simply use $G$.

Hardin (1978) observed that $G$ depends on the current stress state, expressed by the mean effective stress $p$, on the current void ratio $e$, and on the previous stress history experienced by the soil, simply represented by the overconsolidation ratio OCR $=\sigma_{\mathrm{vmax}}^{\prime} / \sigma_{\mathrm{v}}^{\prime}$. He proposed the following form:

$$
\frac{G}{p_{\mathrm{a}}}=S f(e)\left(\frac{p}{p_{\mathrm{a}}}\right)^{n} \mathrm{OCR}^{k}
$$

where $f(e)$ is an empirically defined decreasing function of the void ratio; $p_{\mathrm{a}}$ is the atmospheric pressure (adopted as reference stress); and $S, n$ and $k$ are dimensionless experimentally determined parameters. Following this relationship, a number of authors (e.g. Ishihara, 1982; Jamiolkowski et al., 1994) have provided alternative expressions for the function $f(e)$ for soils of various compositions and index properties. It is worth noting that, in most engineering applications on sands, the soil does not experience a significant variation of the void ratio or of the OCR, the latter parameter also being unknown in most cases. This leads to the use of simplified expressions for the initial shear stiffness, and for the corresponding bulk stiffness:

$$
\begin{aligned}
\frac{G}{p_{a}} & =g\left(\frac{p}{p_{a}}\right)^{n} \\
\frac{K}{p_{a}} & =k\left(\frac{p}{p_{a}}\right)^{n}
\end{aligned}
$$

where $g$ and $k$ are dimensionless constants. The possibility that the exponent $n$ could be different for the bulk and shear moduli might be considered, but is not pursued further here, as this leads to considerable additional complexity in the mathematical development.

In the case of clay soils, further experimental observations and their interpretation (e.g. Houlsby \& Wroth, 1991; Viggiani, 1992; Rampello et al., 1994) have indicated that, for isotropic stress conditions, the small-strain shear stiffness can be expressed as a function of two out of the three variables $e, p$ and OCR. In particular, equation (1) is altered to

$$
\frac{G}{p_{\mathrm{a}}}=S^{*}\left(\frac{p}{p_{\mathrm{a}}}\right)^{n^{*}} R^{k^{*}}
$$

where $R$ is the overconsolidation ratio defined in terms of mean effective stress $R=p_{\mathrm{c}} / p$, with $p_{\mathrm{c}}$ being the mean preconsolidation pressure. This expression can be rearranged as follows:

$$
\frac{G}{p_{\mathrm{a}}}=S^{*}\left(\frac{p}{p_{\mathrm{a}}}\right)^{n^{*}-k^{*}}\left(\frac{p_{\mathrm{c}}}{p_{\mathrm{a}}}\right)^{k^{*}}
$$

showing that the exponent of the mean effective stress $n^{*}$ in equation (4) differs from $n$ in equation (2): some care is therefore necessary in comparing expressions from different authors.

Fewer observations of the small-strain shear stiffness of soils have been performed under anisotropic stress conditions (e.g. Ni, 1987; Hardin \& Blandford, 1989; Jamiolkowski et al., 1994; Rampello et al., 1997; Jovičić \& Coop, 1998). These works suggest that, under anisotropic stress conditions, a modification to those expressions developed entirely from isotropic stress conditions is necessary. For example, Ni (1987) and Hardin \& Blandford (1989) propose the following empirical expression for sands:

$$
\frac{G}{p_{\mathrm{a}}}=S_{i j} f(e) \frac{\left(\sigma_{i} \sigma_{j}\right)^{n / 2}}{p_{\mathrm{a}}^{n}} \mathrm{OCR}^{k}
$$

in which $\sigma_{i}$ and $\sigma_{j}$ are the principal stresses in the plane in which $G$ is measured. (However, the inclusion of the factor $S_{i j}$, which could in principle be a function of the stress ratio, renders this approach, potentially rather complex).

Rampello et al. (1997) proposed an expression for clays that is a modification of equation (4), based on experimental results obtained on a reconstituted clay compressed along radial stress paths characterised by different values of the stress ratio $\eta=q / p$ : 


$$
\frac{G}{p_{\mathrm{a}}}=S_{\eta}^{*}\left(\frac{p}{p_{\mathrm{a}}}\right)^{n^{*}} R_{\eta}^{k^{*}}
$$

where $R_{\eta}$ is the overconsolidation ratio defined with respect to the anisotropic compression line. They suggested that $S_{\eta}^{*}$ could be related to the corresponding value under isotropic stress conditions by the empirical relationship

$$
\frac{S_{\eta}^{*}}{S^{*}}=\exp \left[\frac{n^{*}}{\lambda}\left(N-N_{\eta}\right)\right]
$$

in which $\lambda$ is the slope of the virgin compression line, and $N$ and $N_{\eta}$ are the specific volumes at the reference mean pressure on the isotropic and anisotropic virgin compression lines respectively. In this case, the experimental observations do not establish unequivocally whether the observed variations should be ascribed to the current anisotropic stress state, or to the anisotropic stress history, or to both these components.

In conclusion, the empirical interpretations of the observed small-strain shear moduli quoted above indicate three main features that should be included in the description of the reversible behaviour of soils:

(a) the non-linear dependence on current stress state

(b) the effect of anisotropic stress state

(c) at least for clays, the role of the previous stress history experienced by the material.

Note that if this last effect is introduced, then this corresponds to the concept of elastic-plastic coupling, which is discussed below in the section 'An extended hyperelastic potential for clays'.

\section{LINEAR AND NON-LINEAR ISOTROPIC \\ HYPERELASTICITY}

The evidence cited above indicates that modelling the stiffness of soils must take into account the stress level. However, in some ways appreciation of the implications of these data for theoretical modelling lags behind the empirical knowledge. It is not sufficient simply to adopt arbitrary expressions for the moduli as functions of stress, as this leads to predictions of non-conservative behaviour. Although this can be avoided by adopting a hyperelastic approach, inevitably this leads to a certain additional complexity in the necessary mathematical treatment. This is considered amply justified because of the reassurance it provides that the resulting behaviour is thermodynamically acceptable. We present here an approach that achieves this objective with the minimum of complexity.

A necessary and sufficient condition for the First Law of Thermodynamics to be obeyed for an elastic material is that the stresses can be derived from a free energy (or elastic strain energy) potential. The following will focus on potentials that are expressed as functions of invariants of the strain or of the stress tensor, so that the material behaviour described will be fundamentally isotropic, although it will be seen below that 'stress-induced' anisotropy is predicted under certain conditions.

Expressed in terms of variables appropriate for the triaxial test, the elastic strain energy (internal energy or Helmholtz free energy) $F$ is written as a function of the strains: that is, $F=F(v, \varepsilon)$. It then follows that

$$
\begin{aligned}
& p=\frac{\partial F}{\partial v} \\
& q=\frac{\partial F}{\partial \varepsilon}
\end{aligned}
$$

and further that the tangent bulk and shear moduli are defined by

$$
\begin{aligned}
& K=\frac{\partial p}{\partial v}=\frac{\partial^{2} F}{\partial v^{2}} \\
& 3 G=\frac{\partial q}{\partial \varepsilon}=\frac{\partial^{2} F}{\partial \varepsilon^{2}}
\end{aligned}
$$

Furthermore, it can be shown that off-diagonal terms may in general appear in the incremental stiffness matrix:

$$
\left[\begin{array}{l}
\mathrm{d} p \\
\mathrm{~d} q
\end{array}\right]=\left[\begin{array}{cc}
K & J \\
J & 3 G
\end{array}\right]\left[\begin{array}{l}
\mathrm{d} v \\
\mathrm{~d} \varepsilon
\end{array}\right]
$$

where

$$
J=\frac{\partial p}{\partial \varepsilon}=\frac{\partial q}{\partial v}=\frac{\partial^{2} F}{\partial v \partial \varepsilon}
$$

In the case where $J$ is non-zero the material behaves incrementally in an anisotropic manner, even though $F$ is an isotropic function of the strains. This is a case of stressinduced anisotropy.

Although elastic behaviour can be derived by differentiation, as in equations (9)-(14), this has certain disadvantages. The resulting expressions for the moduli are in terms of the strains, which can be inconvenient, because moduli expressed as functions of stress are usually of more practical use. It is therefore useful to take the Legendre transform of the Helmholtz free energy function to obtain the complementary energy function, or (negative) Gibbs free energy function $E$ :

$$
E=(p v+q \varepsilon)-F
$$

When $E$ is expressed as a function of the stresses, $E=$ $E(p, q)$, the strains may be derived as

$$
\begin{gathered}
v=\frac{\partial E}{\partial p} \\
\varepsilon=\frac{\partial E}{\partial q}
\end{gathered}
$$

and the terms in the compliance matrix

$$
\left[\begin{array}{l}
\mathrm{d} v \\
\mathrm{~d} \varepsilon
\end{array}\right]=\left[\begin{array}{ll}
c_{1} & c_{3} \\
c_{3} & c_{2}
\end{array}\right]\left[\begin{array}{l}
\mathrm{d} p \\
\mathrm{~d} q
\end{array}\right]
$$

can be shown to be

$$
\begin{aligned}
& c_{1}=\frac{3 G}{3 K G-J^{2}}=\frac{\partial v}{\partial p}=\frac{\partial^{2} E}{\partial p^{2}} \\
& c_{2}=\frac{K}{3 K G-J^{2}}=\frac{\partial \varepsilon}{\partial q}=\frac{\partial^{2} E}{\partial q^{2}} \\
& c_{3}=\frac{-J}{3 K G-J^{2}}=\frac{\partial v}{\partial q}=\frac{\partial \varepsilon}{\partial p}=\frac{\partial^{2} E}{\partial p \partial q}
\end{aligned}
$$

The free energy and complementary energy expressions for linear elasticity are both quadratic in form:

$$
\begin{aligned}
& F=p_{a}\left(\frac{k}{2} v^{2}+\frac{3 g}{2} \varepsilon^{2}\right) \\
& E=\frac{1}{p_{a}}\left(\frac{1}{2 k} p^{2}+\frac{1}{6 g} q^{2}\right)
\end{aligned}
$$

with $k$ and $g$ dimensionless constants. From the above it is straightforward to derive $p=k p_{\mathrm{a}} v, q=3 g p_{\mathrm{a}} \varepsilon, K=k p_{\mathrm{a}}$, $G=g p_{\mathrm{a}}$ and $J=0$.

The expressions that give non-linear elasticity (i.e. $K \propto$ $p^{\mathrm{n}}$ ) under purely isotropic stress conditions (i.e. without the 
$q$ and $\varepsilon$ terms) can also be established unambiguously. For $n$ $\neq 1$ the expressions for $F$ and $E$ must be

$$
\begin{aligned}
& F=\frac{p_{a}}{k(2-n)}[k(1-n) v]^{(2-n) /(1-n)} \\
& E=\frac{p^{2-n}}{p_{\mathrm{a}}^{1-n} k(1-n)(2-n)}
\end{aligned}
$$

from either of which one can derive

$$
k(1-n) v=\left(\frac{p}{p_{\mathrm{a}}}\right)^{1-n}
$$

and

$$
\frac{K}{p_{\mathrm{a}}}=k\left(\frac{p}{p_{\mathrm{a}}}\right)^{n}=k[k(1-n) v]^{n /(1-n)}
$$

For $n=1$ the above expressions become singular. A difficulty also arises in the $n=1$ case that, if the volumetric strain is taken as zero at $p=0$, then it is infinite for all finite stresses. This problem can be avoided by shifting the reference point for zero volumetric strain from the origin $(p=0)$ to $p=p_{\mathrm{a}}$. This is achieved by changing equations (24) and (25) to

$$
F=\frac{p_{\mathrm{a}}}{k(2-n)}\left[k(1-n) v^{*}\right]^{(2-n) /(1-n)}
$$

where

$$
v^{*}=v+\frac{1}{k(1-n)}
$$

and

$$
E=\frac{p^{2-n}}{p_{\mathrm{a}}^{(1-n)} k(1-n)(2-n)}-\frac{p}{k(1-n)}
$$

This modifies equation (26) to

$$
1+k(1-n) v=\left(\frac{p}{p_{\mathrm{a}}}\right)^{(1-n)}
$$

and equation (29) to

$$
\frac{K}{p_{\mathrm{a}}}=k\left(\frac{p}{p_{\mathrm{a}}}\right)^{n}=k\left[k(1-n) v^{*}\right]^{n /(1-n)}
$$

but note that this does not affect the expression for stiffness in terms of pressure. The asymptotic expressions for $n=1$ are

$$
\begin{aligned}
& F=\frac{p_{\mathrm{a}}}{k} \exp (k v) \\
& E=\frac{p}{k}\left[\ln \left(\frac{p}{p_{\mathrm{a}}}\right)-1\right]
\end{aligned}
$$

from either of which one can derive

$$
\frac{p}{p_{\mathrm{a}}}=\exp (k v)
$$

and

$$
K=k p
$$

Equations (22) and (23) apply for $n=0$ for any triaxial stress states, whereas equations (28) and (29) (or equations (32) and (33) for $n=1$ ) apply for the case $n \neq 0$, but only on the isotropic axis. It is our purpose in the following to obtain more general expressions that apply both for any triaxial stress states and for $n \neq 0$, and which reduce to each of the above equations in the appropriate special cases.

This generalisation can be done in a variety of ways. We first consider (for simplicity) the case where the reference point for volumetric strain is at $p=0$. Three possible ways of generalising equations (24) and (25) are as follows:

(a) $F$ is of the form

$$
F=v^{m}\left(A v^{2}+B \varepsilon^{2}\right)
$$

where $A, B$ and $m$ are constants. It can be shown that no simple form of $E$ exists for this case.

(b) $F$ is of the form

$$
F=\left(A v^{2}+B \varepsilon^{2}\right)^{m}
$$

which can be shown to result in $E$ of the form

$$
E=\left(C p^{2}+D q^{2}\right)^{m /(2 m-1)}
$$

(c) $E$ is of the form

$$
E=p^{m}\left(A p^{2}+B q^{2}\right)
$$

It can be shown that no simple form of $F$ exists in this case. Einav \& Puzrin (2004) pursue this form of energy function, and show (following Houlsby, 1985) how it also has the disadvantage that it introduces a limiting stress ratio, implying that some stress states are unattainable. Both the inability to derive the $F$ function and the inaccessibility of certain stress states are significant drawbacks to this form of function.

All the forms described above exhibit a constant Poisson's ratio under isotropic stress conditions, consistent with equations (2) and (3). At least at present the available experimental data are insufficient to distinguish definitively between the above three approaches, so the selection is based on the simplicity of the mathematics. The approach that proves to be the most versatile is $(b)$, so this is adopted in the following.

\section{PROPOSED HYPERELASTIC POTENTIAL}

\section{Triaxial formulation}

Following approach $(b)$ above, the generalisation (of equations (22) and (28)) that we seek for the function $F$ is required to consist of a quadratic function of $v^{*}$ and $\varepsilon$, raised to an appropriate power. Inspection of the forms of equations (22) and (28), followed by some calculation to determine some appropriate constant factors, shows that the required general expression is

$$
\begin{aligned}
F= & \frac{p_{\mathrm{a}}}{k(2-n)}[k(1-n)]^{(2-n) /(1-n)} \\
& \times\left[v^{*^{2}}+\frac{3 g \varepsilon^{2}}{k(1-n)}\right]^{(2-n) /(2-2 n)} \\
= & \frac{p_{\mathrm{a}}}{k(2-n)}\left[k v_{\mathrm{o}}(1-n)\right]^{(2-n) /(1-n)}
\end{aligned}
$$

where

$$
v_{\mathrm{o}}^{2}=v^{*^{2}}+\frac{3 g \varepsilon^{2}}{k(1-n)}
$$

Note that $v^{*}$ is used instead of $v$ to move the origin for volumetric strain to $p=p_{\mathrm{a}}$, for consistency with the $n=1$ case. From the above, the strains and the moduli may be obtained by differentiation, as in equations (9)-(14). The 
resulting expressions for the moduli are in terms of the strains.

It can be shown (after some manipulation) that the complementary energy expression, which is the Legendre transform of the expression in equation (40), is

$$
\begin{aligned}
E= & \frac{1}{p_{\mathrm{a}}^{1-n} k(1-n)(2-n)}\left[p^{2}+\frac{k(1-n)}{3 g} q^{2}\right]^{(2-n) / 2} \\
& -\frac{p}{k(1-n)} \\
= & \frac{p_{\mathrm{o}}^{2-n}}{p_{\mathrm{a}}^{1-n} k(1-n)(2-n)}-\frac{p}{k(1-n)}
\end{aligned}
$$

where

$$
p_{\mathrm{o}}^{2}=p^{2}+\frac{k(1-n) q^{2}}{3 g}
$$

Although equations (40) and (41) may appear complex, it can be seen that they have the basic structure of equations (37) and (38) (allowing for the shift of origin for strain). The particular forms of the functions are chosen so that, after differentiation, the moduli reduce to simple expressions. It follows from the above that

$$
\begin{aligned}
v & =\frac{1}{k(1-n)}\left(\frac{p}{p_{\mathrm{a}}^{1-n} p_{\mathrm{o}}^{n}}-1\right) \\
\varepsilon & =\frac{q}{p_{\mathrm{a}}^{1-n} 3 g p_{\mathrm{o}}^{n}}
\end{aligned}
$$

and

$$
\begin{aligned}
& c_{1}=\frac{1}{k(1-n) p_{\mathrm{a}}^{1-n} p_{\mathrm{o}}^{n}}\left[1-\frac{n p^{2}}{p_{\mathrm{o}}^{2}}\right] \\
& c_{2}=\frac{1}{3 g p_{\mathrm{a}}^{1-n} p_{\mathrm{o}}^{n}}\left[1-\frac{n k(1-n) q^{2}}{3 g p_{\mathrm{o}}^{2}}\right] \\
& c_{3}=-\frac{n p q}{3 g p_{\mathrm{a}}^{1-n} p_{\mathrm{o}}^{n+2}}
\end{aligned}
$$

Some of the above expressions (equations (43), (44), (45) and (46)) are valid for $n=1$ as well as $n \neq 1$, but this is not the case for equations (40), (41) and (42). Noting that for $n=1, p_{\mathrm{o}}=p$, the asymptotic values of the compliances for $n=1$ are

$$
\begin{aligned}
& c_{1}=\frac{1}{k p}\left(1+\frac{k q^{2}}{3 g p^{2}}\right) \\
& c_{2}=\frac{1}{3 g p}
\end{aligned}
$$

and

$$
c_{3}=-\frac{q}{3 g p^{2}}
$$

The asymptotic expressions for $n=1$, replacing equations (40), (41) and (42), are

$$
\begin{aligned}
& E=\frac{p}{k}\left[\ln \left(\frac{p}{p_{\mathrm{a}}}\right)-1\right]+\frac{q^{2}}{6 g p} \\
& F=\frac{p_{\mathrm{a}}}{k} \exp \left(k v+\frac{3 g k \varepsilon^{2}}{2}\right)
\end{aligned}
$$

$$
v=\frac{1}{k} \ln \left(\frac{p}{p_{\mathrm{a}}}\right)-\frac{q^{2}}{6 g p^{2}}
$$

Finally we can note that the stiffnesses are given by $K=$ $c_{2} D, 3 G=c_{1} D$ and $J=-c_{3} D$, where $D=3 k g p_{\mathrm{a}}^{2}\left(p_{\mathrm{o}} / p_{\mathrm{a}}\right)^{2 n}$.

The implications of the above choice of free energy (and hence complementary energy) are as follows:

(a) On the isotropic axis simple modulus expressions may be obtained, and $J=0$. On this axis it is also possible to define the Poisson's ratio $v=(3 k-2 g) /(6 k+2 g)$ (alternatively expressed as $g / k=3(1-2 v) / 2(1+v))$. For more general stress points the expressions for the moduli are more complex, but the most important feature is that the moduli are still power functions of the mean stress (although they also depend on the stress ratio). The fact that $J \neq 0$ for general stress states implies stress-induced anisotropic elastic behaviour.

(b) The shapes of shear strain and volumetric strain contours are given directly by equations (42), (43) and (48). Within the range of stress ratios of interest, the volumetric strain contours are similar (but not identical to) parabolae symmetric about the $p$-axis and convex towards the origin. Shear strain contours are curves, convex upwards in the region of the $(p, q)$ plot accessible for reasonable soil properties. For $n=1$ the shear strain contours become straight lines radiating from the origin.

(c) Some undesirable features of the model proposed by Houlsby (1985) and extended by Borja et al. (1997), in particular the crossing of volumetric strain contours, are absent.

Figure 1 shows contours of shear and volumetric strains for selected values of $n$. Note that the volumetric strain contours correspond to undrained stress paths for elastic behaviour. For $n \neq 0$ the approximately parabolic undrained stress paths indicate that (other than on the isotropic axis) the response of the soil is incrementally anisotropic. This stress-induced anisotropy arises as a natural consequence of the hyperelastic formulation, and corresponds well to observations of soil behaviour. Many studies (as cited above) have presented evidence that the stiffness of sands can be expressed as a power function of stress level, but a special feature of the hyperelastic approach adopted here is that it predicts the related effect of the curvature of strain contours. Fig. 2 is reproduced from Shaw \& Brown (1988), who follow the approach of Pappin \& Brown (1980) in plotting 'resilient' shear and volumetric strain contours derived from an extensive series of cyclic tests on granular material. Importantly they show that the volumetric strain contours are approximately parabolic, and curved approximately as in Figs 1(c) or 1(d). The volumetric strain contours (which Shaw \& Brown assume to be straight) are also very similar to a case intermediate between Fig. 1(c) and 1(d). Comparable data for clays were presented by Borja et al. (1997).

\section{General stress formulation}

The results described above can be generalised to other than triaxial stress states, if the free energy $F$ is written as a function of the strains $\varepsilon_{i j}$ and the complementary energy $E$ as a function of the stresses $\sigma_{i j}$. In this case the following expressions must be used:

$$
\begin{aligned}
& E=\sigma_{i j} \varepsilon_{i j}-F \\
& \sigma_{i j}=\frac{\partial F}{\partial \varepsilon_{i j}}
\end{aligned}
$$




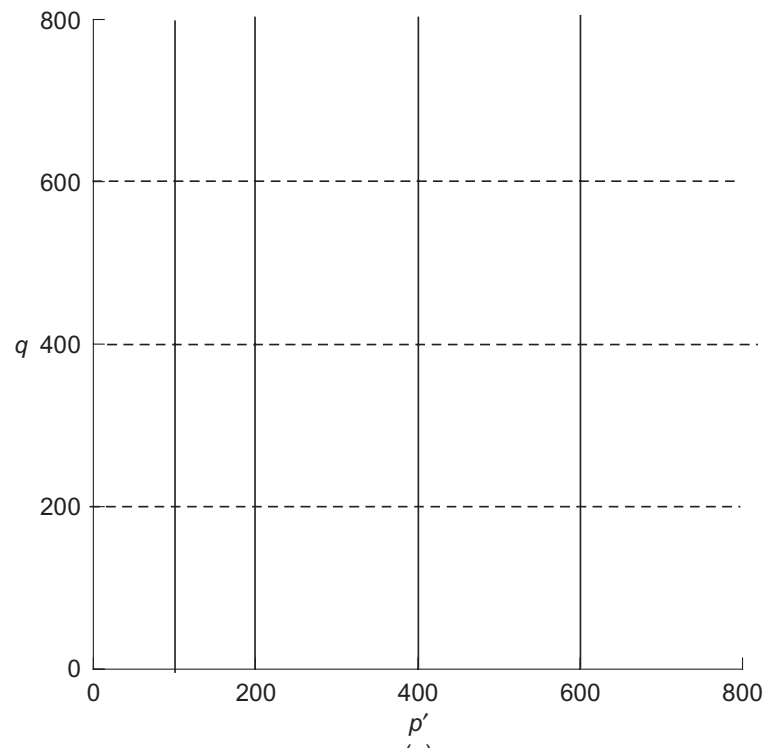

(a)

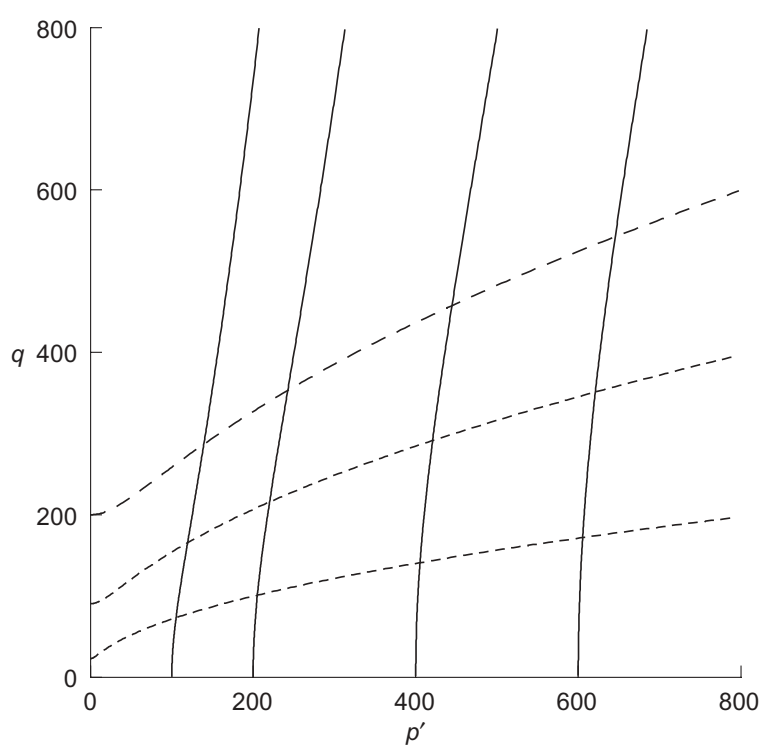

(c)

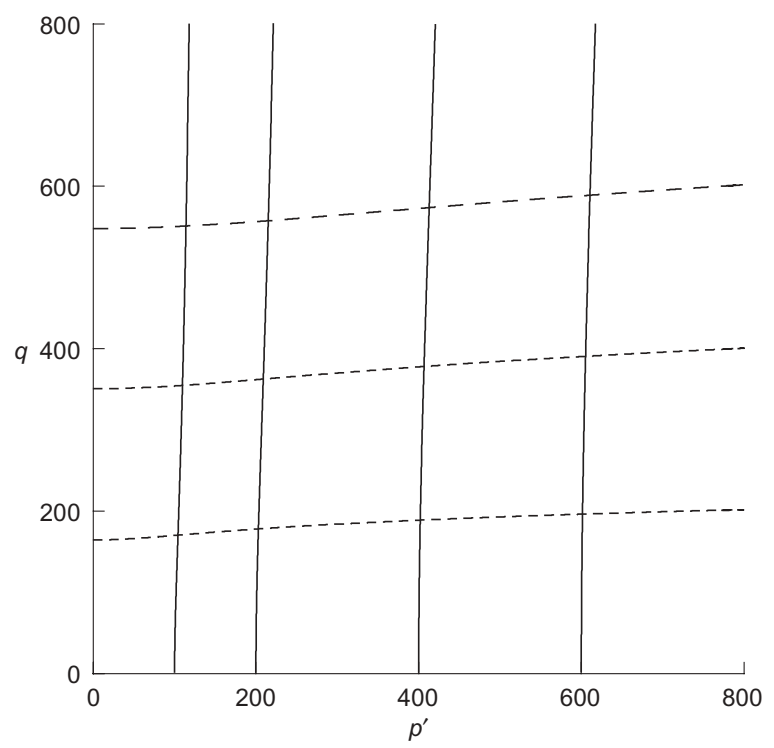

(b)

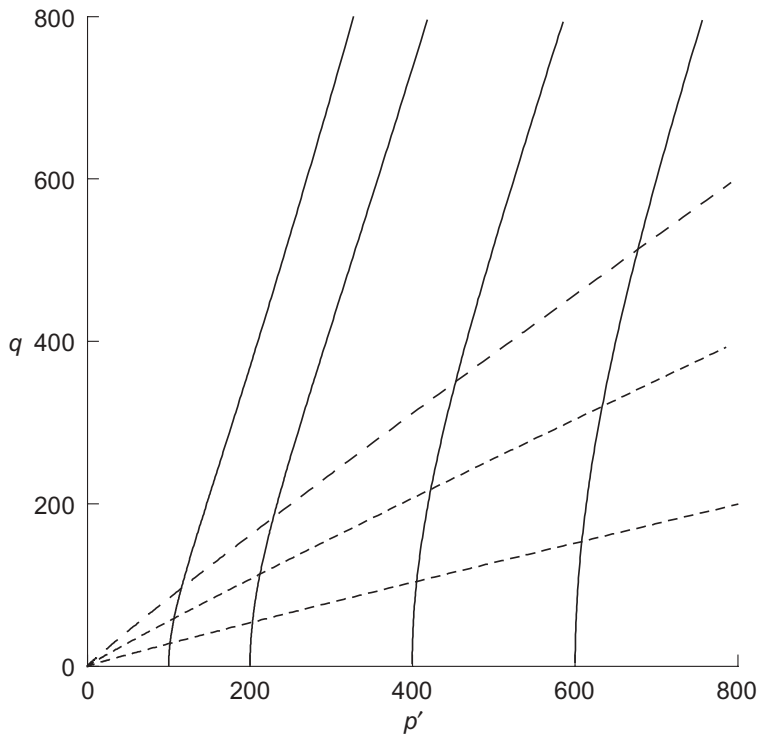

(d)

Fig. 1. Example volumetric and shear strain contours for different values of the exponent $n$ : (a) $n=0 \cdot 0 ;(b) n=0 \cdot 1 ;(c)$ $n=0.5 ;$ (d) $n=0.95$

$$
\varepsilon_{i j}=\frac{\partial E}{\partial \sigma_{i j}}
$$

The stiffness matrix may be obtained as

$$
d_{i j k l}=\frac{\partial \sigma_{i j}}{\partial \varepsilon_{k l}}=\frac{\partial F}{\partial \varepsilon_{i j} \partial \varepsilon_{k l}}
$$

and the compliance matrix as

$$
c_{i j k l}=\frac{\partial \varepsilon_{i j}}{\partial \sigma_{k l}}=\frac{\partial E}{\partial \sigma_{i j} \partial \sigma_{k l}}
$$

The general form of the proposed free energy (for $n \neq 1$ ) is written as before:

$$
F=\frac{p_{\mathrm{a}}}{k(2-n)}\left[k(1-n) v_{\mathrm{o}}\right]^{(2-n) /(1-n)}
$$

where now we have

$$
v_{\mathrm{o}}^{2}=\left[\varepsilon_{i i}+\frac{1}{k(1-n)}\right]\left[\varepsilon_{j j}+\frac{1}{k(1-n)}\right]+\frac{2 g e_{i j} e_{i j}}{k(1-n)} .
$$

The complementary energy for $n \neq 1$ is as before:

$$
E=\frac{p_{\mathrm{o}}^{2-n}}{p_{\mathrm{a}}^{1-n} k(1-n)(2-n)}-\frac{\sigma_{k k}}{3 k(1-n)}
$$

where

$$
p_{\mathrm{o}}^{2}=\frac{\sigma_{m m} \sigma_{n n}}{9}+\frac{k(1-n) s_{m n} s_{m n}}{2 g} .
$$

For $n=1$ the complementary energy is

$$
E=\frac{\sigma_{i i}}{3 k}\left[\ln \left(\frac{\sigma_{j j}}{3 p_{\mathrm{a}}}\right)-1\right]+\frac{3 s_{i j} s_{i j}}{4 g \sigma_{k k}}
$$

and the tangent compliance matrix can be written 


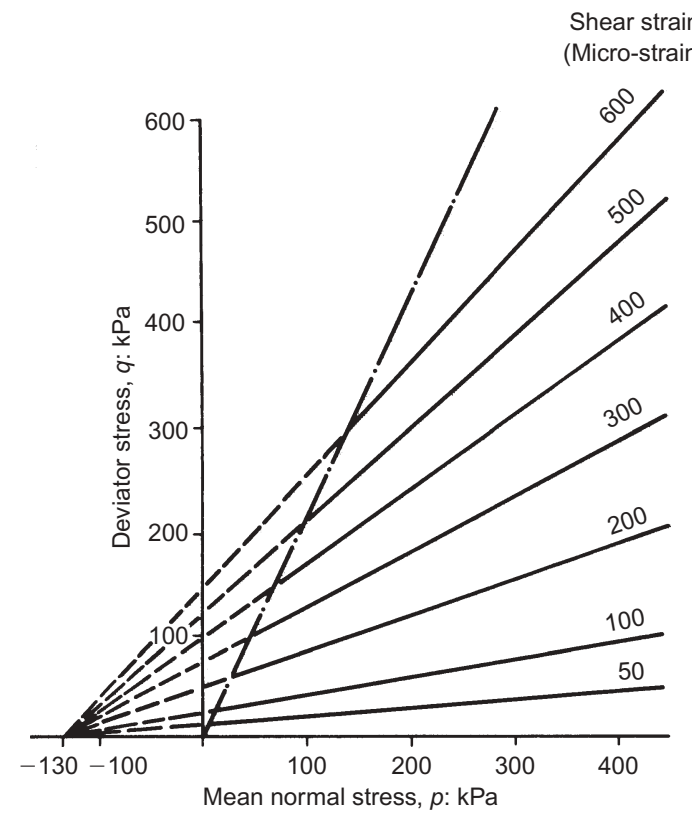

(a)

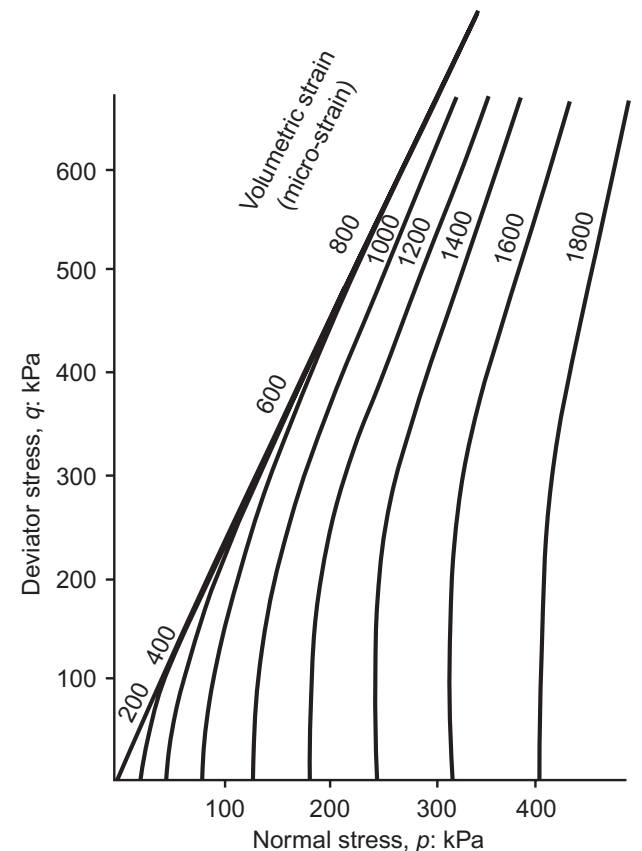

(b)

Fig. 2. (a) Shear and (b) volumetric strain contours presented by Shaw \& Brown (1988), based on experimental data on crushed limestone

$$
\begin{aligned}
c_{i j k l}= & \frac{1}{p_{\mathrm{a}} k(1-n)}\left(\frac{p_{\mathrm{a}}}{p_{\mathrm{o}}}\right)^{n} \\
& \times\left\{\frac{-n}{p_{\mathrm{o}}^{2}}\left[\frac{\sigma_{m m} \delta_{i j}}{9}+\frac{k(1-n) s_{i j}}{2 g}\right]\left[\frac{\sigma_{m m} \delta_{k l}}{9}+\frac{k(1-n) s_{k l}}{2 g}\right]\right. \\
& \left.+\frac{\delta_{i j} \delta_{k l}}{9}+\frac{k(1-n)}{2 g}\left(\delta_{i k} \delta_{j l}-\frac{1}{3} \delta_{k l} \delta_{i j}\right)\right\} \\
= & \frac{1}{p_{\mathrm{a}}}\left(\frac{p_{\mathrm{a}}}{p_{\mathrm{o}}}\right)^{n}\left[\left(\frac{1}{k}+\frac{n s_{m n} s_{m n}}{2 g p_{\mathrm{o}}^{2}}\right) \frac{\delta_{i j} \delta_{k l}}{9}-\right. \\
& \frac{n \sigma_{m m} \times\left(s_{i j} \delta_{k l}+\delta_{i j} s_{k l}\right)+\frac{1}{2 g}\left(\delta_{i k} \delta_{j l}-\frac{1}{3} \delta_{k l} \delta_{i j}\right)}{18 g p_{\mathrm{o}}^{2}} \\
& \left.-\frac{n k(1-n)}{4 g^{2} p_{\mathrm{o}}^{2}} s_{i j} s_{k l}\right]
\end{aligned}
$$

where the latter form is also applicable for $n=1$. It can also be shown that the stiffness matrix can be expressed as

$$
\begin{aligned}
d_{i j k l}= & p_{\mathrm{a}}\left(\frac{p_{\mathrm{o}}}{p_{\mathrm{a}}}\right)^{n}\left[n k \frac{\sigma_{i j} \sigma_{k l}}{p_{\mathrm{o}}^{2}}+k(1-n) \delta_{i j} \delta_{k l}\right. \\
& \left.+2 g\left(\delta_{i k} \delta_{j l}-\frac{1}{3} \delta_{k l} \delta_{i j}\right)\right]
\end{aligned}
$$

It can easily be shown that tests performed in triaxial systems equipped with vertically fitted bender elements allow measurement of the stiffness component $d_{1212}=2 G$, where the vertical and radial directions are 1 and 2 respectively. Under triaxial stress conditions, the above expressions reduce to those obtained in the section 'Triaxial formulation'.

The form of the stiffness matrix in equation (59) has two important consequences. First, and obviously, the terms depend on the stresses (not just the mean stress). This means that the stiffness can be determined only by reference to the complete stress system. Second, and less obviously, it can be shown that (other than on the isotropic axis) the incremental stiffness cannot be expressed just in terms of isotropic stiffnesses: that is, equation (59) does not simply imply stress-dependent values of the parameters $K$ and $G$. The response can be represented only by anisotropic elasticity. This is an example of 'stress-induced' anisotropy: it has nothing to do with the fundamental structure of the material, which is isotropic, but is induced by the stress field. Of course real soils may also exhibit a structural anisotropy. The extension of the concepts discussed here to structurally anisotropic materials is not addressed here.

The compliances or stiffnesses expressed in equations (58) and (59) can be used directly in, for instance, a finite element program for general stress states, ensuring fully conservative elastic behaviour when the moduli are functions of pressure. The expressions require just three dimensionless constants $k, g$ and $n$.

\section{AN EXTENDED HYPERELASTIC POTENTIAL FOR CLAYS}

The proposed complementary energy function does not include any dependence of the reversible behaviour on the internal variables that characterise the hardening of the soil. As already mentioned in the section 'Selected experimental evidence', the elastic stiffness of clays is influenced by the preconsolidation pressure, which plays the role of the hardening variable in the Cam-Clay family of models, and depends on volumetric plastic strain. The following modification of the complementary energy (equation (56)) is proposed to extend it to clay soils:

$$
E=\left(\frac{p_{\mathrm{a}}}{p_{\mathrm{c}}}\right)^{r}\left[\frac{p_{\mathrm{o}}^{2-n}}{p_{\mathrm{a}}^{1-n} k(1-n)(2-n)}-\frac{\sigma_{k k}}{3 k(1-n)}\right]
$$

The introduction of an elastic potential that is dependent on the preconsolidation pressure (i.e. on volumetric plastic strain) results in a behaviour involving the phenomenon of elastic-plastic coupling. In this case great care is required to make a careful distinction between the plastic and irreversi- 
ble components of strain. This issue was first thoroughly addressed for soils by Hueckel (1977) and Maier \& Hueckel (1977). Houlsby (1981) and Collins \& Houlsby (1997) have proposed a thermomechanical framework to describe elasticplastic coupling and its consequences for the overall behaviour of soils.

An important consequence of a plastic-strain-dependent complementary energy relates to the decomposition of the elastic strain tensor. As shown by Hueckel (1977), if

$$
E=E\left(\sigma_{i j}, \varepsilon_{i j}^{\mathrm{p}}\right)
$$

then the 'elastic' component of the strain is

$$
\varepsilon_{i j}^{\mathrm{e}}=\frac{\partial E}{\partial \sigma_{i j}}
$$

leading to the incremental form

$$
\dot{\varepsilon}_{i j}^{\mathrm{e}}=\frac{\partial^{2} E}{\partial \sigma_{i j} \partial \sigma_{k l}} \dot{\sigma}_{k l}+\frac{\partial^{2} E}{\partial \sigma_{i j} \partial \varepsilon_{k l}^{\mathrm{p}}} \dot{\varepsilon}_{k l}^{\mathrm{p}}
$$

Equation (63) shows that the elastic strain increment can be

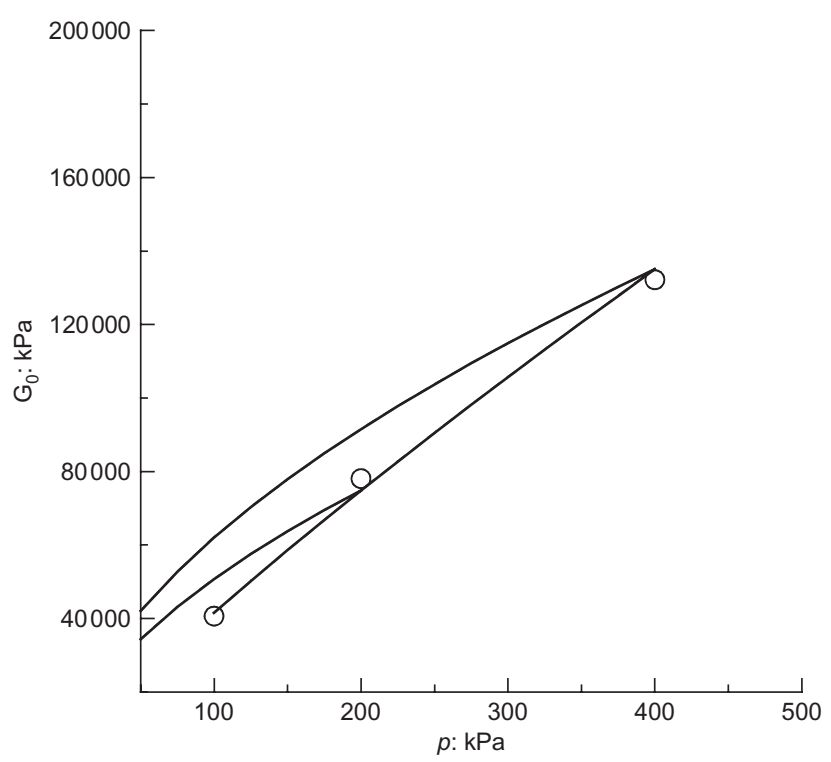

(a)

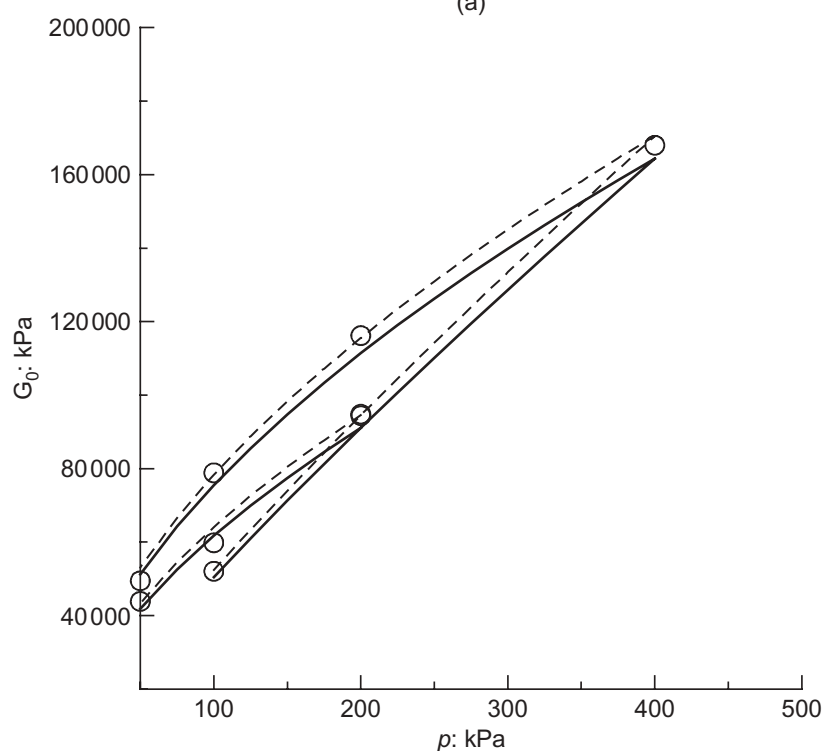

(c) decomposed into a first term defining the reversible strain $\left(\dot{\varepsilon}_{i j}^{\mathrm{r}}\right)$ and a second term called the coupled strain $\left(\dot{\varepsilon}_{i j}^{\mathrm{c}}\right)$. The latter accounts for the change of stiffness as plastic straining occurs, whereas the former expresses the stiffness of the material at fixed plastic strain.

In the following, a comparison is made between predictions obtained by equation (60) and the observed behaviour of a reconstituted clay. The experimental data are from Rampello et al. (1997), and are only briefly summarised here. A series of tests were carried out in computercontrolled triaxial cells, equipped with vertically fitted bender elements. The samples were loaded and unloaded along radial stress paths, defined by values of the stress ratio $\eta=$ $q / p$ of $0.0,0.5$ and 0.7 . During the compression stages, bender element measurements were performed for different values of the effective stress and overconsolidation ratio. Fig. 3 shows the values of $G$ against the mean pressure, using circles for the experimental data, dotted lines for the empirical expression (equation (7)) proposed by Rampello et al. (1997), and continuous lines for the prediction derived from equation (60) and using the set of parameters $k=692$, $g=415, n=0.56, r=0.29$ and the reference pressure $p_{\mathrm{a}}$ $=100 \mathrm{kPa}$. The values of the preconsolidation pressure for

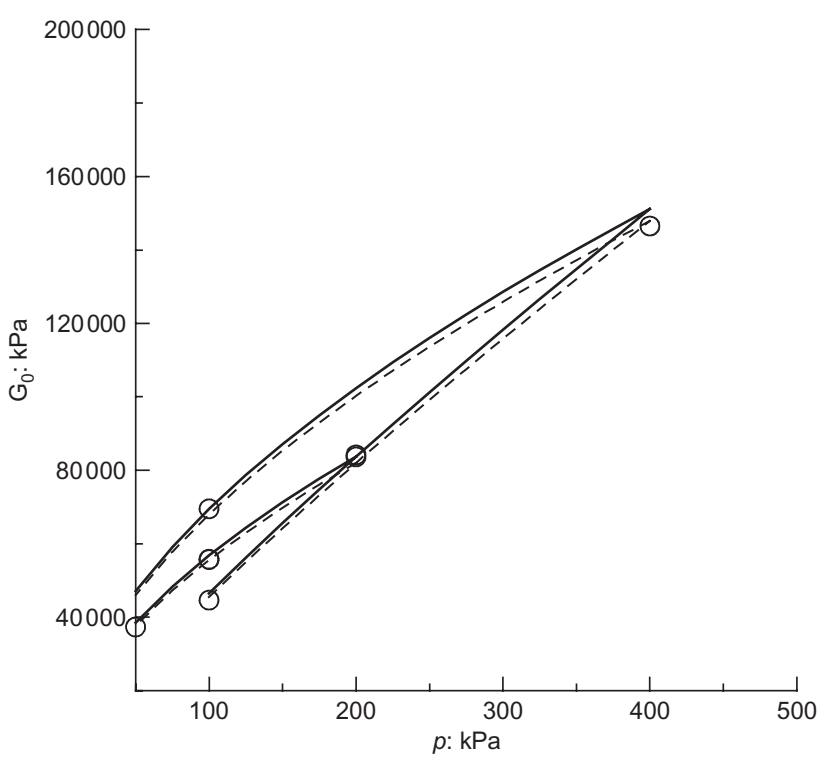

(b)

Fig. 3. Comparison between moduli from equation (60) and data from Rampello et al. (1997): (a) $\eta=0$; (b) $\eta=0 \cdot 5$; (c) $\eta=0 \cdot 7$ 
$\eta>0$ have been derived assuming that the shape of the yield surface is that of the Modified Cam-Clay ellipse with $M=0 \cdot 8$. The comparison between equation (60) and the data is very satisfactory. Note that, by comparing the parameters of equation (7) and those of the proposed potential, it follows that $n=n^{*}-k^{*}, r=k^{*}, g=S_{\eta=0}^{*}$, while $k$ has been estimated assuming that, under isotropic stress conditions, the Poisson's ratio $v$ is equal to $0 \cdot 25$.

Finally, it can be observed that bender element testing on elastic-plastic-coupled soils provides data on the reversible part of the strain $\left(\dot{\varepsilon}_{i j}^{\mathrm{r}}\right)$ rather than on the elastic strain. Each measurement is performed without inducing any variation of the preconsolidation pressure $p_{\mathrm{c}}$ (i.e. $\left.\dot{\varepsilon}_{k l}^{\mathrm{p}}=0\right)$ : thus each individual test gives data on only the first compliance term $\left(\partial^{2} E / \partial \sigma_{i j} \partial \sigma_{k l}\right)$ appearing in the decomposition equation (63).

\section{CONCLUSIONS}

In this paper we have presented a framework based on hyperelasticity for describing the non-linear elastic stiffness of soils as a function of stress. In order to account realistically for the variation of stiffness of soils, stress-dependent small-strain moduli must be adopted. Simplistic models in which tangent moduli are arbitrarily defined as functions of stress can lead to non-conservative response, in violation of the laws of thermodynamics. In contrast, the hyperelastic approach guarantees thermodynamic acceptability. Simple strain and complementary energy potentials are defined that realistically capture the small-strain stiffness of sands and clays. Secondary effects, such as stress-induced anisotropy, are predicted and correspond to empirical observations. An approach such as that described here should be employed in describing the elastic behaviour at the heart of more complex elastic-plastic models.

\author{
NOTATION \\ $A, B, C, D$ constants \\ $c_{1}, c_{2}, c_{3}$ compliances \\ $c_{i j k l}$ compliance matrix \\ $d_{i j k l} \quad$ stiffness matrix \\ $D$ function of stresses \\ $E$ complementary energy (negative Gibbs free energy) \\ $e$ voids ratio \\ $e_{i j}$ deviator of strain tensor \\ $F \quad$ strain energy (Helmholtz free energy) \\ $G$ shear modulus \\ $G_{0} \quad$ small strain shear modulus \\ $g$ shear stiffness factor \\ $J$ coupling modulus \\ $K$ bulk modulus \\ $k$ (1) Overconsolidation ratio exponent (also with * \\ superscript), (2) bulk stiffness factor \\ $M$ slope of critical state line in $p^{\prime}, q$ plot \\ $m$ exponent \\ $N$ specific volume at reference pressure on normal \\ compression line (also with $\eta$ subscript) \\ $n$ pressure exponent (also with $*$ superscript) \\ $O C R$ overconsolidation ratio in terms of vertical stress \\ $p, p^{\prime} \quad$ mean effective stress \\ $p_{a} \quad$ atmospheric pressure \\ $p_{c}$ preconsolidation pressure in terms of mean stress \\ $p_{0}$ function of stresses \\ $q$ deviator stress invariant \\ $R$ overconsolidation ratio in terms of mean effective \\ stress (also with subscript) \\ $r$ preconsolidation pressure exponent \\ $S$ dimensionless stiffness factor (also with * superscript \\ and $\eta$ subscript) \\ $S_{i j} \quad$ anisotropic stiffness factor \\ $s_{i j}$ deviator of effective stress tensor \\ $v$ volumetric strain
}

$v^{*}$ modified volumetric strain

$v_{0} \quad$ function of strains

$\delta_{i j} \quad$ Kronecker- $\delta$ (unit tensor)

$\varepsilon$ shear strain invariant

$\varepsilon_{i j}$ strain tensor

$\varepsilon_{i j}^{c} \quad$ coupled strain tensor

$\varepsilon_{i j}^{e}$ elastic strain tensor

$\varepsilon_{i j}^{p} \quad$ plastic strain tensor

$\varepsilon_{i j}^{r} \quad$ reversible strain tensor

$\eta$ triaxial stress ratio

$\kappa$ slope of swelling line in consolidation plot

$\lambda$ slope of compression line in consolidation plot

$v$ Poisson's ratio

$\sigma_{i}, \sigma_{j}$ principal effective stresses

$\sigma_{i j}$ effective stress tensor

$\sigma_{v}^{\prime} \quad$ vertical effective stress

$\sigma_{v \max }^{\prime}$ vertical preconsolidation stress

\section{REFERENCES}

Borja, R. I., Tamagnini, C. \& Amorosi, A. (1997). Coupling plasticity and energy-conserving elasticity models for clays. J. Geotech. Engng 123, No. 10, 948-956.

Boyce, H. R. (1980). A non-linear model for the elastic behaviour of granular materials under repeated loading. Proceedings of the international symposium on soils under cyclic and transient loading, Swansea, Vol. 1, pp. 285-294.

Collins, I. F. \& Houlsby, G. T. (1997). Application of thermomechanical principles to the modelling of geotechnical materials. Proc. R. Soc. London Ser. A 453, No. 1964, 1975-2001.

Einav, I. \& Puzrin, A. M. (2004). Pressure dependent elasticity and energy conservation in elastoplastic models for soils. J. Geotech. Geoenviron. Engng 130, No. 1, 81-92.

Fung, Y. C. (1965). Foundations of solid mechanics. Englewood Cliffs, NJ: Prentice Hall.

Hardin, B. O. (1978). The nature of stress-strain behaviour for soils. Earthquake engineering and soil dynamics: Proceedings of the ASCE Geotechnical Engineering Division Specialty Conference, Pasadena, pp. 3-90.

Hardin, B. O. \& Blandford, G. E. (1989). Elasticity of particulate materials. J. Soil Mech. Found. Engng Div., ASCE 115, No. 6, $788-805$.

Houlsby, G. T. (1981). A study of plasticity theories and their applicability to soils. $\mathrm{PhD}$ thesis, University of Cambridge.

Houlsby, G. T. (1985). The use of a variable shear modulus in elastic-plastic models for clays. Comput. Geotech. 1, No. 1, $3-13$.

Houlsby, G. T. \& Wroth, C.P. (1991). The variation of the shear modulus of a clay with pressure and overconsolidation ratio. Soils Found. 31, No. 3, 138-143.

Hueckel, T. (1977). The flow law of the granular solids with variable unloading rule. In Problemes de la Rheologie et de Mecanique des Sols (ed. W. K. Nowacki), pp. 203-217. Warsaw: PWN.

Hueckel, T., Tutumluer, E. \& Pellegrini, R. (1992). A note on nonlinear elasticity of isotropic overconsolidated clays. Int. $J$. Numer. Anal. Methods Geomech. 16, No. 8, 603-618.

Ishihara, K. (1982). Evaluation of soil properties for use in earthquakes response analysis. Proceedings of the international symposium on numerical models in geomechanics, Zurich, pp. 237-259.

Jamiolkowski, M., Lancellotta, R. \& Lo Presti, D. C. F. (1994). Remarks on the stiffness at small strain of six Italian clays. Proc. 1st Int. Conf. Pre-failure Deformation Characteristics of Geomaterials, Sapporo 2, 817-836.

Jovičić, V. \& Coop, M. R. (1998). The measurement of the stiffness anisotropy in clays with bender element tests in the triaxial apparatus. ASTM Geotech. Test. J. 21, No. 1, 3-10.

Lade, V. \& Nelson, R. B. (1987). Modelling the elastic behaviour of granular materials. Int. J. Numer. Anal. Methods Geomech. 11, No. 5, 521-542.

Maier, G. \& Hueckel, T. (1977). Non-associated and coupled flow rules of elastoplasticity for geotechnical media. Proc. 9th Int. Conf. Soil Mech. Found. Engng, Tokyo, Speciality session 7, $129-142$.

Ni, S. H. (1987). Dynamic properties of sand under true triaxial 
stress states from resonant column and torsional shear tests. $\mathrm{PhD}$ thesis, The University of Texas, Austin.

Niemunis, A. \& Cudny, M. (1998). On hyperelasticity for clays. Comput. Geotech. 23, No. 4, 221-236.

Pappin, J. W. \& Brown, S. F. (1980). Resilient stress-strain behaviour of a crushed rock. Proceedings of the international symposium on soils under cyclic and transient loading, Swansea, pp. 169-177.

Rampello, S., Silvestri, F. \& Viggiani, G. (1994). The dependence of $G_{0}$ on stress state and history in cohesive soils. Proc. 1st Int. Conf. on pre-failure deformation characteristics of geomaterials, Sapporo 2, 1155-1160.

Rampello, S., Viggiani, G. M. B. \& Amorosi, A. (1997). Small- strain stiffness of reconstituted clay compressed along constant triaxial stress ratio paths. Géotechnique 47, No. 3, 475-489.

Shaw, P. \& Brown, S. F. (1988). Behaviour of granular materials under repeated load biaxial and triaxial stress conditions. Géotechnique 38, No. 4, 627-634.

Vermeer, P. (1978). A double hardening model for sand. Géotechnique 28, No. 4, 413-433.

Viggiani, G. (1992). Small strain stiffness of fine grained soils. $\mathrm{PhD}$ thesis, City University, London.

Zytynski, M., Randolph, M., Nova, R. \& Wroth, C. P. (1978). On modelling the unloading-reloading behaviour of soils. Int. $J$ Numer. Anal. Methods Geomech. 2, 87-93. 